\title{
Kinetic relaxing processes in collective dynamics of pure liquids
}

\author{
T.Bryk, I.Mryglod \\ Institute for Condensed Matter Physics \\ of the National Academy of Sciences of Ukraine, \\ 1 Svientsitskii Str., 79011 Lviv, Ukraine
}

Received August 7, 2004

\begin{abstract}
The role of nonhydrodynamic relaxing processes in collective dynamics of pure liquids is studied within a generalized collective modes approach. A five-variable model of generalized hydrodynamics is solved analytically in long-wavelength limit. Among five collective modes we found three hydrodynamic and two relaxing kinetic excitations. Two latter ones describe the viscous and heat relaxation in liquid. A comparison of analytical results and molecular dynamics data is performed for a Lennard-Jones fluid and a liquid metal. A crossover from standard hydrodynamics to molecular regime in relaxational behaviour of simple liquids is discussed.
\end{abstract}

Key words: generalized hydrodynamics, collective excitation, dynamical structure factor, relaxation processes

PACS: 05.20.Jj,61.20.Ja,61.20.LC

\section{Introduction}

Collective dynamics in liquids is an extremely difficult problem for theoretical treatment because many microscopic processes occur on different time scales. A clear picture of collective excitations and their contributions to experimentally measured quantities (like dynamical structure factor $S(k, \omega)$ with $k$ and $\omega$ being wavenumber and frequency, respectively) is so far well established only in hydrodynamic limit when $k \rightarrow 0, \omega \rightarrow 0[1-3]$. Three hydrodynamic equations reflect in fact the local conservation laws and describe only the dynamical processes on large spatial and temporal scales comparing with average interatomic distance and characteristic molecular time, i.e. when liquids are treated as continuum without any details of atomic structure. Beyond the hydrodynamic region, where there are mostly located the wavenumbers being available in real experiments on light or neutron scattering and in computer molecular dynamics (MD) simulations, the short-time processes with finite lifetime and a spatial scale of several Angstroms become very important and can significantly contribute to the shape of dynamical structure factors. There- 
fore, much effort has been made in order to derive analytical expressions for the hydrodynamic time correlation functions (in particular, for the density-density time correlation function $F_{n n}(k, t)$ simply connected via inverse time-Fourier transform to $S(k, \omega))$ that generalize the known hydrodynamic results and could be used beyond the hydrodynamic region. One of the most successful attempts, a nonlocal mode coupling theory [4], can potentially explain dynamical phenomena in very broad range of temperatures and densities. However, due to the necessity to evaluate intermediate integrations over wavenumbers it can be used practically in a limited number of applications. Thus, less sophisticated schemes of generalized hydrodynamics based on the memory function formalism in the local form [1] are often more useful for practical needs.

However we still observe a lack of clear understanding of the mechanisms of spectra formation in liquids (especially in two- and many-component cases) as well as the role of coupling effects between relaxing processes and propagating excitations. Another important issue is the analysis of contributions from different collective excitations beyond the hydrodynamic region. Many researchers still analyze the data from scattering experiments or MD simulations with wavenumbers accessible far beyond the hydrodynamic region using simple hydrodynamic Landau-Placzek expression [1] for dynamical structure factor. A new tendency in analyzing the experimental data lies in fitting procedure based on an expression for dynamical structure factor found within the framework of memory function approach, in which the second order memory function is modelled as a sum of three exponentials in general case, which reflect one thermal and two viscous, slow and fast, channels of correlation decay [5]. Such an approximation corresponds to a five-pole model of dynamical structure factor, i.e. in terms of eigenmodes the model corresponds to a treatment of collective dynamics by a pair of propagating (sound) modes and three relaxing processes. The authors [5] interpret the three relaxing processes contributing to the central peak of dynamical structure factor as: generalized hydrodynamic process of thermodiffusivity with the generalized coefficient of thermodiffusion $D_{\mathrm{T}}(k)$; a slow process of structural (alpha-)relaxation, and an unspecified fast relaxation process.

In this work we study collective dynamics in pure fluids using an approach of generalized hydrodynamics known as the method of generalized collective modes (GCM) [6,7], in which the collective excitations in liquids are defined as the poles of relevant spectral functions. The main feature of this approach is the existence of the so-called kinetic collective excitations, which have finite lifetime and together with generalized hydrodynamic excitations contribute to the shape of density-density time correlation functions $F_{n n}(k, t)$ beyond the hydrodynamic region. Since the GCM approach takes into account the mode coupling effects in local approximation, i.e. interaction of hydrodynamic and kinetic excitations with the same wavenumber $k$, one can obtain a very precious information about dispersion law and damping of collective excitations studied within an analytical treatment of different models in the GCM approach. Moreover, the GCM time correlation functions can be a good starting point for nonlocal mode coupling approach, because they keep all the information about the local coupling of different microscopic processes. Recently [8] 
we have shown, on an example of mass-concentration fluctuations in binary liquids, very clear physical mechanisms of damping the kinetic propagating excitations by a hydrodynamic relaxing process $[9,10]$. It was concluded, that the damping of the kinetic optic-like excitations in both longitudinal [8] and trasverse [9,10] cases is defined by mutual diffusion and demixing properties, i.e. the binary systems that tend to demixing when the atoms are mainly surrounded by the like ones do not support optic-like excitations. At the same time high mutual diffusion of the components does not permit optic-like excitations to propagate. Another example of propagating kinetic collective excitations can be heat waves, studied for the case of a liquid metal in [11]. Regarding relaxing collective excitations we have shown $[11,12]$, that the shape of the density-density time correlation functions in the range of wavenumbers close to the main peak position of the static structure factor $S(k)$ is almost completely defined by a single relaxing kinetic mode originated by relaxation of the cage of nearest neighbors.

In this study we are interested in the origin of relaxing kinetic modes in the long-wavelength region. We shall analytically solve a five-variable dynamical model in the long-wavelength limit in order to understand the behaviour of the kinetic longwavelength relaxing processes. It is not an easy task to correctly separate purely real eigenvalues obtained numerically within the GCM scheme in a small- $k$ region. That is why the knowledge of analytical expressions for kinetic relaxing modes would be helpful. In particular, analytical solutions provide us with deeper understanding a crossover from hydrodynamic to molecular-scale region, when the main contributions to the central peak of dynamical structure factor $S(k, \omega)$ essentially change.

The remaining paper is organized as follows: in the next Section we give some general remarks on the GCM approach and details of our MD simulations; in Section III a five-variable model for pure liquids is solved analytically in long-wavelength limit that permits to study two solutions corresponding to kinetic relaxing processes; for the wavenumbers beyond hydrodynamic region we report a numerical GCM analysis of collective dynamics in a Lennard-Jones fluid and liquid metallic Beryllium; and in the last Section we give the conclusions of this study.

\section{Method}

The approach of GCM allows us to study fast dynamical processes on the same footing with hydrodynamic processes. In this study we use the following five-variable basis set of dynamical variables for generalized treatment of longitudinal collective dynamics in pure liquids:

$$
\mathbf{A}^{(5)}(k, t)=\left\{n(k, t), J^{\mathrm{L}}(k, t), \dot{J}^{\mathrm{L}}(k, t), h(k, t), \dot{h}(k, t)\right\},
$$

where the number density $n(k, t)$, longitudinal mass-current $J^{\mathrm{L}}(k, t)$ and heat density $h(k, t)$ are the hydrodynamic variables, while extended ones contain overdots which mean time derivative of relevant fluctuations. The basis set of five variables (1) is used in constructing a generalized hydrodynamic matrix $\mathbf{T}(k)$ [7], eigenvalues $z_{j}(k)$ of which represent dynamical eigenmodes existing on different spatial scales reflected 
by the wavenumber $k$ in the studied liquid. Purely real eigenvalues correspond to relaxing processes (we will mark them as $d_{j}(k)$ to distinguish them from the complex eigenvalues $\left.z_{j}(k)=\sigma_{j}(k) \pm \mathrm{i} \omega_{j}(k)\right)$, while complex roots reflect propagating processes in the liquid. An important consequence of GCM treatment is the possibility to represent any time correlation function between basis dynamical variables as a sum over mode contributions. For example, the symmetrized form for normalized densitydensity time correlation functions within the GCM approach reads:

$$
\frac{F_{n n}^{\mathrm{GCM}}(k, t)}{S(k)}=\sum_{j}^{N_{\mathrm{r}}} A_{n n}^{j} \mathrm{e}^{-d_{j}(k) t}+\sum_{j}^{N_{\mathrm{p}}} \mathrm{e}^{-\sigma_{j}(k) t}\left[B_{n n}^{j} \cos \left(\omega_{j} t\right)+C_{n n}^{j} \sin \left(\omega_{j} t\right)\right] .
$$

Here in our case of the five-variable dynamical model $N_{\mathrm{r}}+2 N_{\mathrm{p}}=5$, where $N_{\mathrm{r}}$ and $N_{\mathrm{p}}$ are the numbers of relaxing modes and pairs of complex-conjugated eigenvalues, respectively. The expression (2) generalizes the three-term hydrodynamic expression [13] onto the case of additional kinetic modes in the liquid. The amplitudes of mode contributions from relaxing processes $A_{n n}^{j}(k)$, as well as symmetric $B_{n n}^{j}(k)$ and asymmetric amplitudes $C_{n n}^{j}(k)$ from propagating processes are the functions of wavenumber and will be studied here numerically for two pure liquids.

We performed MD simulations of a system of 1000 particles in microcanonical ensemble for a Lennard-Jone fluid at reduced density $n^{*}=0.845$ and at temperature $T^{*}=1.71$ and a liquid metal Be with mass density $\rho=1688.3 \mathrm{~kg} \cdot \mathrm{m}^{-3}$ and temperature $T=1560 \mathrm{~K}$. The time evolution of all five dynamical variables was observed over the production run of $3 \cdot 10^{5}$ timesteps and stored for the calculations of relevant time correlation functions and static averages needed for estimation of the $5 \times 5$ generalized hydrodynamic matrix $\mathbf{T}^{(5)}(k)$. Eigenvalues of the generalized hydrodynamic matrix formed the spectra of eigenmodes $d_{j}(k)$ and $z_{j}(k)$, and were used in the analysis of density-density time correlation functions according to expression (2).

\section{Results and discussion}

\subsection{Analytical solutions in long-wavelength region}

In the long-wavelength limit the five-variable model (1) can be solved analytically. This generalized five-variable dynamical model has been known in liquid theory since the paper by Akcasu and Daniels [14], in which they studied generalized transport coefficients in pure liquids. However, in the literature there is no analytical solution for this five-variable dynamical model in terms of generalized collective modes. The matrix elements of generalized hydrodynamic matrix were estimated in the following way [7]:

$$
\mathbf{T}^{(5)}(k)=\mathbf{F}(k, t=0) \tilde{\mathbf{F}}^{-1}(k, z=0),
$$

where the $5 \times 5$ matrices of time correlation functions $\mathbf{F}(k, t)$ and their Laplace transforms $\tilde{\mathbf{F}}(k, z)$ were constructed using dynamical variables (1). Analytical expressions for the hydrodynamic correlation times $\tau_{i j}(k), i, j=n, h$ in $k \rightarrow 0$ limit were taken from the paper [13]. Only the terms within the precision not higher than 
$O\left(k^{2}\right)$ were kept in $\mathbf{T}^{(5)}(k)$, which reads:

$$
\mathbf{T}^{(5)}(k)=\left(\begin{array}{ccccc}
0 & -i \frac{k}{m} & 0 & 0 & 0 \\
0 & 0 & -1 & 0 & 0 \\
T_{31} & T_{32} & d_{2}^{0} & T_{34} & T_{35} \\
0 & 0 & 0 & 0 & -1 \\
T_{51} & T_{52} & T_{53} & T_{54} & d_{3}^{0}
\end{array}\right)
$$

where

$$
d_{2}^{0}=\frac{c_{\infty}^{2}-c_{\mathrm{s}}^{2}}{D_{\mathrm{L}}}, \quad d_{3}^{0}=\frac{c_{\mathrm{V}}}{m \lambda}\left(G^{\mathrm{h}}-\frac{(\gamma-1)}{\kappa_{\mathrm{T}}}\right) .
$$

In the last expressions $c_{\infty}$ and $c_{\mathrm{s}}$ are the high-frequency and adiabatic speed of sound, respecively, $c_{\mathrm{V}}$ is the specific heat at constant volume, $\lambda$ is thermal conductivity, $\kappa_{\mathrm{T}}$ is isothermal compressibility, $\gamma$ is ratio of specific heats, and $G^{\mathrm{h}}$ is heat rigidity modulus. The other matrix elements read:

$$
\begin{aligned}
& T_{31}=-\mathrm{i} k \frac{m}{\gamma} c_{\mathrm{s}}^{2} d_{2}^{0}, \quad T_{32}=k^{2} \frac{1}{\gamma}\left(c_{\mathrm{s}}^{2}+2 \Gamma d_{2}^{0}\right), \\
& T_{34}=-\mathrm{i} k \frac{\gamma-1}{\alpha_{\mathrm{T}} T} d_{2}^{0}, \quad T_{35}=-\mathrm{i} k \frac{\gamma-1}{\alpha_{\mathrm{T}} T c_{\mathrm{s}}^{2}}\left(c_{\mathrm{s}}^{2}+\left(D_{\mathrm{L}}-D_{\mathrm{T}}\right) d_{2}^{0}\right), \\
& T_{51}=-k^{2} \frac{m \alpha_{\mathrm{T}} T c_{\mathrm{s}}^{2}\left(D_{\mathrm{L}}-D_{\mathrm{T}}\right)}{\gamma D_{\mathrm{L}}} D_{\mathrm{T}} d_{3}^{0}, \quad T_{52}=-\mathrm{i} k \frac{\alpha_{\mathrm{T}} T c_{\mathrm{s}}^{2}}{\gamma} d_{3}^{0}, \\
& T_{53}=-\mathrm{i} k \alpha_{\mathrm{T}} T\left(\frac{c_{\mathrm{s}}^{2}}{\gamma}+\frac{\left(D_{\mathrm{L}}-D_{\mathrm{T}}\right) D_{\mathrm{T}}}{D_{\mathrm{L}}} d_{3}^{0}\right), \quad T_{54}=k^{2} \frac{2 \Gamma D_{\mathrm{T}}}{D_{\mathrm{L}}} d_{3}^{0},
\end{aligned}
$$

where $2 \Gamma=D_{\mathrm{L}}+(\gamma-1) D_{\mathrm{T}}$ with $D_{\mathrm{L}}$ and $D_{\mathrm{T}}$ being kinematic viscosity and thermal diffusivity, respectively, and $\alpha_{\mathrm{T}}$ is the linear expansion coefficient.

The set of eigenmodes for this model contains three hydrodynamic modes

$$
d_{1}(k)=D_{\mathrm{T}} k^{2}, \quad z_{ \pm}(k)=\Gamma k^{2} \pm \mathrm{i} c_{\mathrm{s}} k
$$

exactly as they appear in hydrodynamic approach being thermodiffusive relaxing process $d_{1}(k)$ and propagating sound excitations $z_{ \pm}(k)$. Additionaly two purely real eigenvalues correspond to two kinetic relaxing modes:

$$
d_{2}(k)=d_{2}^{0}-D_{\mathrm{L}} k^{2}+(\gamma-1) \Delta k^{2}, \quad d_{3}(k)=d_{3}^{0}-\gamma D_{\mathrm{T}} k^{2}-(\gamma-1) \Delta k^{2} .
$$

In (7) the following shortcut was used:

$$
\Delta=\frac{d_{2}^{0} d_{3}^{0}}{d_{3}^{0}-d_{2}^{0}} \frac{D_{\mathrm{T}}}{D_{\mathrm{L}} c_{\mathrm{s}}^{2}}\left(D_{\mathrm{T}}-D_{\mathrm{L}}\right)^{2}
$$

The last terms in the right hand sides of (7) appear due to the coupling between the heat and density fluctuations. When the coupling is not taken into account, i.e. $\gamma=1$, one would obtain results of viscoelastic approximation. The expressions (7) reveal that the modes $d_{2}(k)$ and $d_{3}(k)$ reflect viscous and heat relaxation in liquids. 
The most interesting fact is the increasing lifetime of kinetic relaxing processes $d_{2}(k)$ and $d_{3}(k)$, i.e. inversed eigenvalue, against wavenumbers. The tendency is opposite to the behaviour of the lifetime for hydrodynamic processes of thermal diffusivity and sound propagation, and at the boundary of hydrodynamic region these relaxation times can have comparable values. This implies that the approximate location of the crossover region can be estimated from the condition of equivalent relaxation times for hydrodynamic and kinetic processes. In the simplest case when we neglect the coupling between thermal and viscous processes, i.e. $\gamma=1$, one can obtain that the crossover between hydrodynamic thermodiffusive relaxation and structural one takes place at

$$
k_{\mathrm{cr}} \approx\left[\frac{\left(c_{\infty}^{2}-c_{\mathrm{s}}^{2}\right)}{D_{\mathrm{L}}\left(D_{\mathrm{L}}+D_{\mathrm{T}}\right)}\right]^{1 / 2} .
$$

This expression means that in highly viscous liquids $k_{\text {cr }}$ becomes extremely small and structural processes are more important on large spatial scales than in regular liquids.

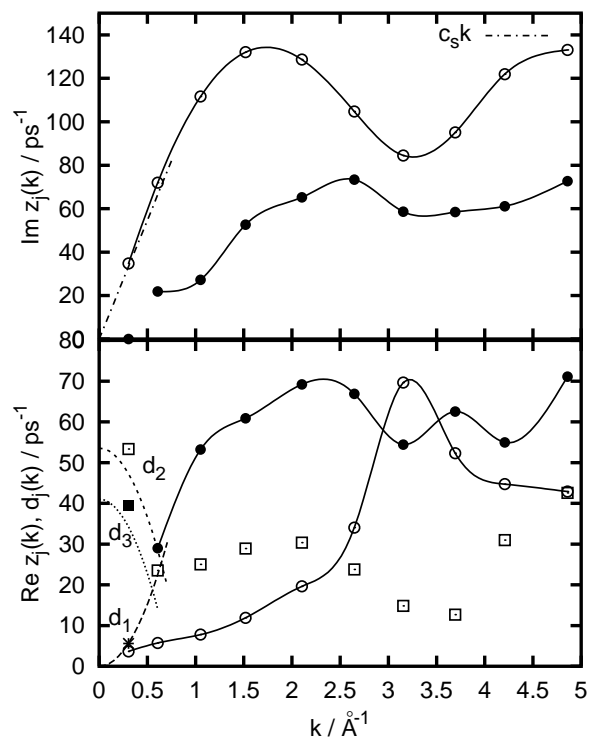

Figure 1. Spectrum of dynamical eigenmodes in liquid metallic Be at $1560 \mathrm{~K}$. In the top frame the dispersion of generalized sound excitations (open circles) and low-frequency heat waves (filled circles) is shown together with hydrodynamic linear sound dispersion. In the lower frame the damping coefficients of propagating excitations (relevant circles) are shown together with relaxing modes $d_{j}(k)$. Analytical results for relaxing processes are shown by dashed and dotted lines.

\subsection{Numerical analysis beyond hydrodynamic region}

Analytical treatment of the five-variable model (1) is possible only in the longwavelength limit, while numerically we can estimate all matrix elements of the generalized hydrodynamic matrix $\mathbf{T}^{(5)}(k)$ at any $k$-point sampled in MD simulations. 
The results for five eigenmodes obtained for liquid Be at $1560 \mathrm{~K}$ are shown in figure 1 . From the top frame of figure 1 one can note that in general there can exist two pairs of propagating excitations in the metallic liquid:

(i) generalized sound excitations (open circles) with almost linear dispersion law in the long-wavelength region and roton-like minimum in the region of wavenumbers close to the location of the main peak of static structure factor $k_{\mathrm{p}} \approx$ $3.4 \AA^{-1}$,

(ii) and kinetic heat waves (filled circles), which can exist only behind the so-called propagation gap.

The linear dispersion $c_{\mathrm{s}} k$ with the hydrodynamic speed of sound $c_{\mathrm{s}}=10935 \mathrm{~m} / \mathrm{s}$ obtained from the exptrapolation to $k=0$ of generalized ratio $\gamma(k) / S(k)$ shows, that the smallest point sampled in MD is quite close to the hydrodynamic region. The damping coefficients of propagating excitations are shown in the lower frame by line-connected circles. Three relaxing processes are shown as well in the lower frame of figure 1 by the following symbols: $d_{1}(k)$ by asterisks, $d_{2}(k)$ by open boxes and $d_{3}(k)$ by filled boxes. The dashed and dotted lines in small- $k$ region correspond to the analytical expressions (6) and (7) with the thermodynamic quantities obtained by us from the numerical GCM analysis: $D_{\mathrm{T}}=60.9 \AA^{2} / \mathrm{ps}, D_{\mathrm{L}}=67.6 \AA^{2} / \mathrm{ps}$ and $c_{\infty}=12482 \mathrm{~m} / \mathrm{s}$. Remarkably, that in agreement with our analytical treatment the kinetic relaxing modes $d_{2}(k)$ and $d_{3}(k)$ obtained by numerical approach (symbols) show a decaying behaviour in the small- $k$ region. At the boundary of propagation gap, two relaxing modes connected to thermal processes $d_{1}(k)$ and $d_{3}(k)$ disapper creating a pair of heat waves. The kinetic relaxing process $d_{2}(k)$, which is defined by longitudinal viscosity in a small- $k$ region, becomes the slowest process behind the hydrodynamic region. As it was shown in $[11,12]$ this kinetic relaxing process corresponds to the effects of structural relaxation in pure liquid. Note, that at $k=0$ analytical expressions (7) for kinetic relaxing modes give very reasonable agreement with the numerical values obtained at $k_{\min }$ by numerical GCM analysis. Also, the crossections of lines reflecting analytical results in the small- $k$ region exactly correspond to the width of propagatin gap for heat waves, and crossover region $k_{\mathrm{cr}}(8)$.

In figure 2 we show, for the case of liquid Be, the mode contributions to the normalized dynamical structure factor, which in GCM approach can be represented as follows:

$$
\begin{aligned}
\frac{S(k, \omega)}{S(k)}= & \sum_{j}^{N_{\mathrm{r}}} A_{n n}^{j} \frac{d_{j}(k)}{\omega^{2}+d_{j}^{2}(k)}+\sum_{j}^{N_{\mathrm{p}}} B_{n n}^{j}\left[\frac{\sigma_{j}(k)}{\left(\omega+\omega_{j}(k)\right)^{2}+\sigma_{j}^{2}(k)}\right. \\
& \left.+\frac{\sigma_{j}(k)}{\left(\omega-\omega_{j}(k)\right)^{2}+\sigma_{j}^{2}(k)}\right]+C_{n n}^{j}\left[\frac{\omega+\omega_{j}(k)}{\left(\omega+\omega_{j}(k)\right)^{2}+\sigma_{j}^{2}(k)}\right. \\
& \left.-\frac{\omega-\omega_{j}(k)}{\left(\omega-\omega_{j}(k)\right)^{2}+\sigma_{j}^{2}(k)}\right] .
\end{aligned}
$$

Relaxing processes which define the central peak of $S(k, \omega)$ are characterized by the amplitudes $A_{n n}^{j}(k)$. In a small- $k$ region there exist three contributions from the 


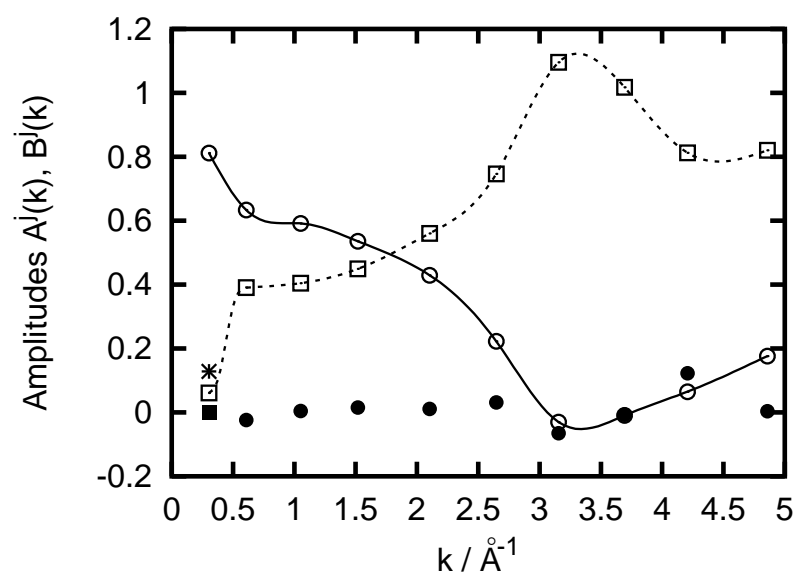

Figure 2. Amplitudes of main contributions to the dynamical structure factor (9) from the dynamical eigenmodes in liquid Be obtained by five-variable GCM approach. The symbols correspond to contributions of the same eigenmodes as in previous figure.

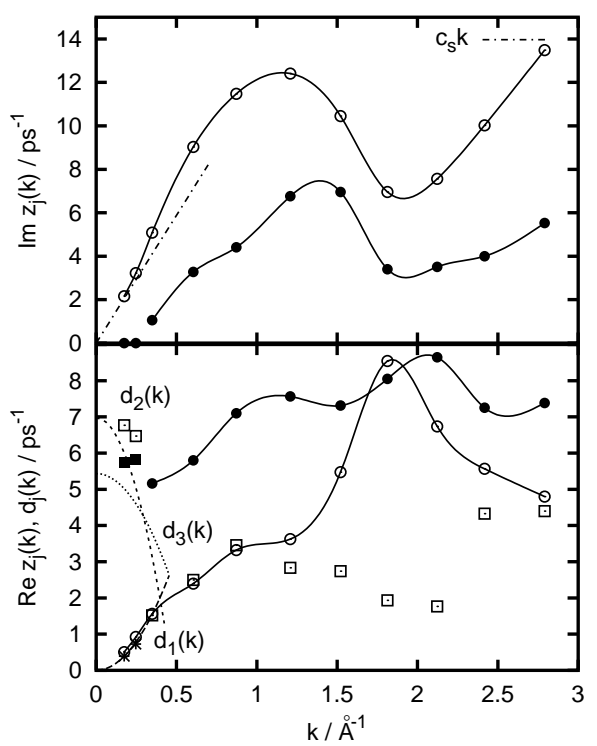

Figure 3. Spectrum of dynamical eigenmodes in Lennard-Jones fluid at reduced density $n^{*}=0.845$ and temperature $T^{*}=1.71$. The symbols and lines correspond to the same modes as in figure 1 .

relaxing processes: one from the hydrodynamic process of thermal diffusion, which according to hydrodynamic treatment should be $(\gamma-1) / \gamma$ in the limit $k \rightarrow 0$, and two kinetic processes, which contributions must vanish in hydrodynamic limit. We estimated the value of $\gamma=1.15 \pm 0.05$ for liquid Be, which means that $A_{n n}^{1}(k \rightarrow$ $0) \approx 0.13$ is in good agreement with the figure 2 . The contribution from sound excitations tends to the value $1 / \gamma$ in long-wavelength limit again in good agreement with hydrodynamic predictions. More interesting is behaviour of contribution from the kinetic relaxing mode $d_{2}(k)$ which describes processes of structural relaxation. 
From figure 2 one can see that for $k>0.5 \AA^{-1}$ the shape of the central peak of $S(k, \omega)$ is almost completely defined by the contribution from the kinetic relaxing process $d_{2}(k)$. The crossover in contributions to the central peaks of $S(k, \omega)$ which we obtained in the analytical GCM treatment takes place around $k_{\mathrm{cr}} \approx 0.35 \AA^{-1}$.

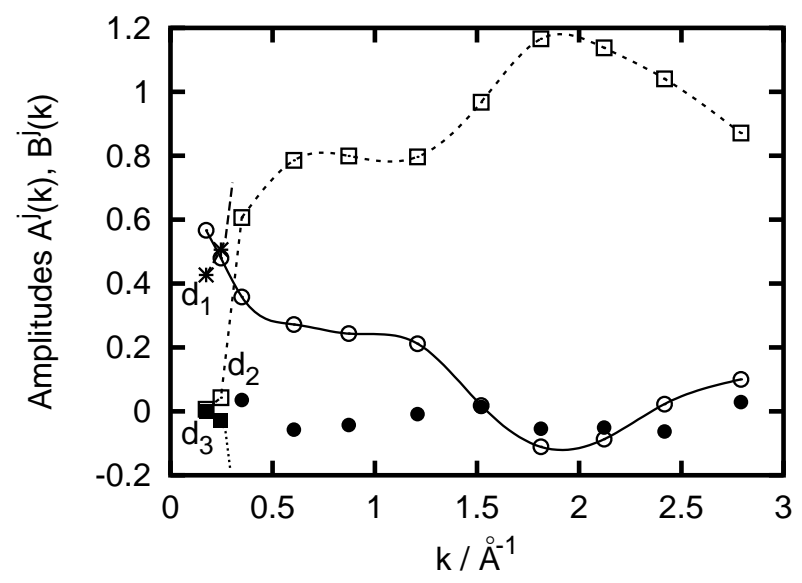

Figure 4. Amplitudes of main contributions to the dynamical structure factor (9) from the dynamical eigenmodes in a Lennard-Jones liquid obtained by fivevariable GCM approach. The symbols correspond to contributions of the same eigenmodes as in previous figure.

Similar behaviour of eigenmodes against the wavenumber is observed for LennardJones liquid. In figure 3 we show the propagating and relaxing dynamical eigenvalues along with analytical results for $d_{2}(k)$ and $d_{3}(k)$ obtained in $k \rightarrow 0$ limit. We note again, that the analytical treatment predicts correctly the width of propagation gap for heat waves and crossover region $k_{\text {cr }}$ where the change in main contributions to the central peak of dynamical structure factor takes place. The thermodynamic quantities obtained by us from the numerical GCM analysis have the following values: $D_{\mathrm{T}}=12.7 \AA^{2} / \mathrm{ps}, D_{\mathrm{L}}=16.2 \AA^{2} / \mathrm{ps}, c_{\mathrm{s}}=1177 \mathrm{~m} / \mathrm{s}, c_{\infty}=1789 \mathrm{~m} / \mathrm{s}$, and $\gamma=1.64$.

In figure 4 the amplitudes $A_{n n}^{j}(k)$ and $B_{n n}^{j}(k)$ of main contributions to the dynamical structure factor $S(k, \omega)(9)$ for the Lennard-Jones fluid are shown. An essential difference here comparing to the case of liquid $\mathrm{Be}$ is in the values of the amplitudes from hydrodynamic excitations, which due to larger ratio of specific heats $\gamma$ tend to the following values: $A_{n n}^{1}(k \rightarrow 0) \approx 0.39$ and $B_{n n}^{1}(k \rightarrow 0) \approx 0.61$. Due to wider region with almost hydrodynamic behaviour of the lowest collective excitations one can observe the specific behaviour of amplitudes $A^{1}(k)$ and $A^{3}(k)$ which by approaching the boundary gap for heat waves (in our case approximately at $k \approx 0.3 \AA^{-1}$ ) display divergence to $+/-\infty$, respectively. Such a behaviour of amplitudes of relaxing processes can be easily obtained analytically using relevant two-variable model. The other amplitudes behave quite similar as in the case of liquid Be with the leading contributions from the generalized sound excitations $\left(B_{n n}^{1}(k)\right.$ - open circles in figure 4$)$ and relaxing kinetic mode responsible for structural relaxation $\left(A_{n n}^{2}(k)\right.$ - open boxes in figure 4) to the shape of $S(k, \omega)$ far beyond the hydrodynamic region. 


\section{Conslusions}

The main consclusions of this study are as follows:

(i) we have solved analytically a generalized five-variable model of longitudinal collective dynamics in long-wavelength limit for pure liquids. It was shown that two eigenvalues additional to the hydrodynamic ones correspond to kinetic relaxing processes in the liquid with the increasing lifetime against $k$. The kinetic relaxing processes correspond to structural and heat rigidity relaxations in the liquid;

(ii) the behaviour of relaxing long-wavelength eigenvalues obtained from the numerical study of dynamical eigenmodes in a wide region of wavenumbers is in agreement with analytical results;

(iii) the behaviour of amplitudes of mode contributions to the central peak of dynamical structure factor $S(k, \omega)$ reveals a crossover between hydrodynamic contribution from thermal diffusion to leading contribution from structural relaxation beyond the hydrodynamic region.

\section{References}

1. Boon J.-P., Yip S. Molecular Hydrodynamics. New-York, McGraw-Hill, 1980.

2. March N.H., Tosi M.P. Atomic Dynamics in Liquids. London, Macmillan Press, 1976.

3. Hansen J.-P., McDonald I.R. Theory of Simple Liquids. London, Academic, 1986.

4. Bosse J., Götze W., Lücke M. // Phys. Rev. A, 1978, vol. 17, p. 434.

5. Scopigno T., Balucani U., Ruocco G., Sette F. // J. Phys.: Cond. Matt., 2000, vol. 12, p. 8009.

6. de Schepper I.M., Cohen E.G.D., Bruin C., van Rijs J.C., Montfrooij W., de Graaf L.A. // Phys. Rev. A, 1988, vol. 38, p. 271.

7. Mryglod I.M., Omelyan I.P., Tokarchuk M.V. // Mol. Phys., 1995, vol. 84, p. 235.

8. Bryk T., Mryglod I. // J. Phys.: Cond. Matt., 2002, vol. 14, p. L445.

9. Bryk T., Mryglod I. // J. Phys.: Cond. Matt., 2000, vol. 12, p. 6305.

10. Bryk T., Mryglod I. // Phys. Rev. E, 2000, vol. 62, p. 2188.

11. Bryk T., Mryglod I. // Phys. Rev. E, 2001, vol. 63, p. 051202; vol. 64, p. 032202.

12. Bryk T., Mryglod I. // J. Phys.: Cond. Matt., 2001, vol. 13, p. 1343.

13. Cohen C., Sutherland J.W.H., Deutch J.M. // Phys. Chem. Liq., 1971, vol. 2, p. 213.

14. Akcasu A.Z., Daniels E. // Phys. Rev. A, vol. 2, p. 962. 


\title{
Кінетичні релаксаційні процеси в колективній динаміці простих рідин
}

\author{
Т.Брик, І.Мриглод \\ Інститут фізики конденсованих систем НАН України, \\ 79011 Львів, вул. Свєнціцького, 1 \\ Отримано 7 серпня 2004 р.
}

Негідродинамічні релаксаційні процеси в простих рідинах досліджуються в рамках підходу узагальнених колективних мод. Аналітично розв'язано п'ятизмінну модель узагальненої гідродинаміки в довгохвильовій границі. Серед п'яти отриманих власних мод $€$ три гідродинамічні та дві кінетичні моди, які описують процеси в'язкої та теплової релаксації в рідині. Порівняння аналітичних результатів з даними, що отримані в комп'ютерних симуляціях методом молекулярної динаміки, проведено для Ленард-Джонсівської рідини та рідкого метала. Обговорюється кросовер у релаксаційній поведінці рідин від гідродинамічної області до молекулярних масштабів.

Ключові слова: узагальнена гідродинаміка, колективне збудження, динамічний структурний фактор, релаксаційні процеси

PACS: 05.20.Jj,61.20.Ja,61.20.LC 
